\title{
Empfehlungen zur Diagnose und Behandlung der chronischen Herzinsuffizienz
}

\author{
Arbeitsgruppe für Herzinsuffizienz der Schweizerischen Gesellschaft für Kardiologie
}

1 Bemerkung der Autoren: Die männliche Form «Patient» steht auch stellvertretend für die weibliche Form «Patientin».

Redaktionskomitee

W. Kiowski, Zürich; P. Dubach, Chur; P. Mohacsi, Bern; A. F. Gallino, Bellinzona; O. Bertel, Zürich; R. Lerch, Genf; P. Rickenbacher, Basel; G. Noll, Zürich; O. M. Hess, Bern.

Als Vertreter der Schweizerischen Gesellschaft für Innere Medizin und der Schweizerischen Gesellschaft für Allgemeinmedizin haben

D. Conen, Aarau, und P. Périat, Basel, mitgewirkt.

\section{Korrespondenz:}

Prof. Dr. med. Otto M. Hess Schweizer Herz- und Gefässzentrum Kardiologie

Inselspital

CH-3010 Bern

Tel. 0316329653

Fax 0316324771

E-Mail: otto.martin.hess@insel.ch
Die Prävalenz der chronischen Herzinsuffizienz in den westlichen Industrieländern beträgt zwischen 0,4 und 2\%. Entsprechend gibt es zurzeit in der Schweiz etwa 100000 Patienten ${ }^{1}$ mit einer Herzinsuffizienz [1]. Die Prävalenz steigt mit zunehmendem Alter an. Pro Jahr erkranken in den USA etwa 400000 Personen neu an chronischer Herzinsuffizienz, in der Schweiz etwa 20000. Während noch vor etwa 3-4 Jahrzehnten die Hypertonie und valvuläre Herzkrankheiten die häufigsten Ursachen der chronischen Herzinsuffizienz darstellten, ist heute die koronare Herzkrankheit mit ungefähr 70\% die häufigste zugrundeliegende Erkrankung. Trotz grosser Fortschritte beim Verständnis der Pathophysiologie und darauf basierend der Therapie der chronischen Herzinsuffizienz ist die Morbidität und Mortalität dieses Syndroms unverändert gross und beansprucht einen zunehmenden Teil der finanziellen Ressourcen des Gesundheitswesens. In Anbetracht der medizinischen und ökonomischen Bedeutung dieses Syndroms ist deshalb eine korrekte Diagnosestellung und adäquate Therapie wichtig. Die vorliegenden Empfehlungen basieren auf den Richtlinien der Arbeitsgruppe Herzinsuffizienz der Europäischen Gesellschaft für Kardiologie [2]. Bei den Therapieempfehlungen sind die neueren Erkenntnisse aufgrund kontrollierter Untersuchungen berücksichtigt, soweit diese zum Zeitpunkt der Abfassung der Empfehlungen publiziert waren. Entsprechend wurden für die Therapieempfehlungen 3 Evidenzgrade angegeben, die in Tabelle 1 definiert sind.

\section{Definition}

Herzinsuffizienz ist ein progressives, klinisches Syndrom, welches durch verschiedene kardiale Erkrankungen, die zur Verminderung der Pumpfunktion führen, verursacht wird. Die Diagnose stützt sich auf Symptome der Herzinsuffizienz (Müdigkeit, Atemnot in Ruhe oder unter Belastung, Ödeme, s. Tab. 2) und objektive Befunde der kardialen Dysfunktion (Verminderung der Auswurffraktion usw.). Obwohl die Beeinträchti- gung der Myokardfunktion bei der Entwicklung der Herzinsuffizienz initial im Vordergrund steht, kommt es im weiteren Verlauf zu einer verminderten Durchblutung der Skelettmuskulatur und der Nieren, verbunden mit der Aktivierung neuro-humoraler Systeme. Diese Erkenntnisse haben manche Autoren dazu veranlasst, die neuro-humorale Stimulation in die Definition der chronischen Herzinsuffizienz miteinzubeziehen. Bei Verdacht auf Herzinsuffizienz ist es wichtig, die Diagnose auszuschliessen oder zu bestätigen. Dazu bieten sich neben der klinischen Untersuchung verschiedene nicht-invasive und invasive Methoden an.

Die häufigste Ursache einer Herzinsuffizienz ist die koronare Herzkrankheit (60-70\%), gefolgt in $20-30 \%$ von der hypertensiven Herzkrankheit, zu 5-10\% von den Kardiomyopathien, in 3-10\% von den valvulären Herzkrankheiten und in $1-2 \%$ von den kongenitalen Herzerkrankungen. Oft finden sich beim gleichen Patienten 2 oder mehrere dieser Ursachen. Anämie, Toxine oder Schilddrüsenfunktionsstörung können eine Herzinsuffizienz auslösen oder begünstigen. Alkohol kann zur Alkoholkardiomyopathie führen und als Co-Faktor in der Pathophysiologie der dilatativen Kardiomyopathie bzw. hypertensiven Herzerkrankung eine wichtige Rolle spielen.

Verschiedene Einteilungen der Herzinsuffizienz [2] sind im Gebrauch. Neben der akuten und der chronischen Form sind vor allem die Rechts- bzw. Linksherzinsuffizienz, das LowOutput- und das High-Output-Syndrom, die Ruhe- bzw. Belastungsinsuffizienz oder die mechanische bzw. metabolische Form der Herzinsuffizienz unterschieden worden. Eine wichtige Unterscheidung bezieht sich auf die systolische bzw. diastolische Dysfunktion, die sowohl prognostisch als auch therapeutisch von Bedeutung ist.

Die systolische Dysfunktion wird charakterisiert durch:

1. Befunde und/oder Symptome der Herzinsuffizienz;

2. Befunde der verminderten Pumpfunktion (verringerte Auswurffraktion bzw. vergrössertes Kammervolumen). 
Tabelle 1

Definition der Evidenzgrade.

Evidenzgrad A belegt durch multiple randomisierte Studien oder Metaanalysen

Evidenzgrad B belegt durch eine randomisierte Studie oder mehrere nicht-randomisierte Studien

Evidenzgrad C basierend auf Konsens von Experten

Häufigste Ursache der systolischen Dysfunktion ist der Zustand nach Herzinfarkt mit regional verminderter Pumpfunktion und LV-Remodeling mit Dilatation des linken Ventrikels.

Die diastolische Dysfunktion wird charakterisiert durch:

1. Befunde oder Symptome der Herzinsuffizienz;

2. Befunde des erhöhten linksventrikulären Füllungsdruckes mit den Zeichen der verminderten Kammerfüllung;

3. normale oder leicht verminderte systolische Pumpfunktion.

\section{Klinik der Herzinsuffizienz}

\section{Anamnese}

Die Anamnese kann wichtige Hinweise auf das Voliegen einer Herzinsuffizienz geben, indem die Symptome wie Müdigkeit, Atemnot in Ruhe oder unter Belastung, Orthopnoe, nächtliche
Dyspnoe, Beinödeme, Nykturie oder Rhythmusstörungen für die Herzinsuffizienz typisch sind. Das Vorliegen einer koronaren Herzkrankheit mit Zustand nach Herzinfarkt oder einer arteriellen Hypertonie kann Hinweise auf die Ätiologie der Erkrankung geben.

\section{Klinische Untersuchung}

Ein erhöhter Halsvenendruck, ein positiver hepato-jugulärer Reflux, das Vorliegen eines 3. Herztones, feuchte Rasselgeräusche über den basalen Lungenfeldern, eine Hepatomegalie, Knöchelödeme und allenfalls Aszites sind alleine oder in Kombination Hinweise auf eine Herzinsuffizienz (Tab. 2). Es sollte jedoch berücksichtigt werden, dass viele dieser Befunde nicht spezifisch für eine Herzinsuffizienz sind (z.B. Hepatomegalie, Aszites oder Knöchelödeme), und die klinische Erfassung dieser Zeichen bei älteren und adipösen Patienten sowie bei Frauen häufig schwierig ist. Eine bereits eingeleitete Therapie kann diese Befunde verbessern, so dass eine schwere Herzinsuffizienz mit deutlicher symptomatischer Einschränkung auch ohne diese klinischen Zeichen vorliegen kann. Die Kombination der oben erwähnten Befunde bei einem Patienten mit Symptomen der Herzinsuffizienz weist allerdings eine hohe Spezifität auf, die Sensitivität dieser Befunde ist oft jedoch gering.

Tabelle 2

Klinische Diagnose der Herzinsuffizienz gemäss WHO (Concise Guide to the Management of Heart Failure).

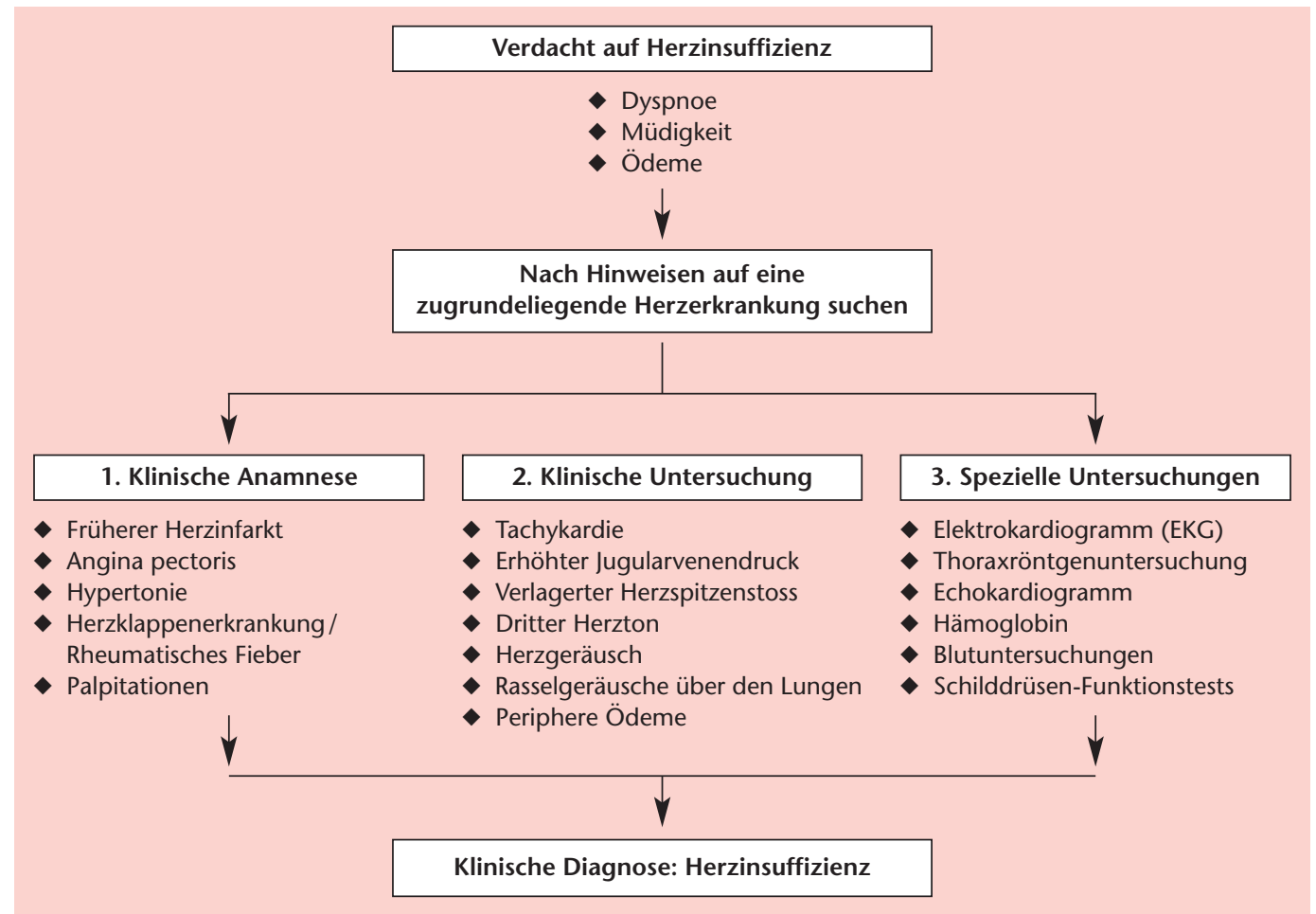


Tabelle 3

Funktionelle Einteilung von Herzpatienten (Richtlinien der New York Heart Association).

\begin{tabular}{ll} 
Klasse I & keine Beschwerden selbst bei grosser Anstrengung \\
\hline Klasse II & Beschwerden bei grösseren Anstrengungen (z. B. Treppensteigen, Bergaufgehen) \\
\hline Klasse III & Beschwerden bei kleineren Anstrengungen (z. B. in der Ebene gehen, Hausarbeiten) \\
\hline Klasse IV & Beschwerden bei kleinsten Anstrengungen und/oder in Ruhe
\end{tabular}

Tabelle 4

Maximale Sauerstoffaufnahme unter Belastung nach Weber et al. [3].

\begin{tabular}{lll} 
Funktionelle Beeinträchtigung & $\mathrm{VO}_{2} \max .(\mathrm{ml} / \mathrm{kg} / \mathrm{min})$ & $\%$ max. Soll \\
\hline keine & $>18$ & $>80$ \\
\hline leicht & $14-18$ & $65-80$ \\
\hline mässig & $10-14$ & $50-65$ \\
\hline schwer & $<10$ & $<50$ \\
\hline (Für Laufband: $+10 \%$ für $\mathrm{VO}_{2}$ max) & &
\end{tabular}

Als funktionelle Einteilung wird heute die Klassifikation nach der New York Heart Association (NYHA-Klassifizierung) gewählt (Tab. 3).

\section{Nicht-invasive Diagnostik}

EKG

Patienten mit chronischer Herzinsuffizienz haben selten ein völlig normales EKG. Das EKG gibt Aufschluss über den Rhythmus, und das Vorliegen von Q-Zacken (zusammen mit einer entsprechenden Anamnese) kann auf einen durchgemachten transmuralen Herzinfarkt hinweisen. Überleitungsstörungen, intraventrikuläre Reizleitungsstörungen und Zeichen der Linksund Rechtshypertrophie sowie Veränderungen der ST- und T-Segmente sind häufig aber unspezifisch und wenig sensitiv bezüglich möglicher Ursachen (Myokarditis, Perikarditis, Ischämie).

\section{Thoraxröntgen}

Das Thoraxröntgenbild, wenn möglich im dorso-ventralen und lateralen Strahlengang aufgenommen, kann Aufschluss über die Grösse des Herzens, weniger zuverlässig über die Grösse der einzelnen Herzkammern, das Vorliegen einer pulmonal-arteriellen Hypertonie und Pleuraergüsse, Verkalkungen von Herzklappen, des Myokards und des Perikards geben.

\section{Hämatologie und Blutchemie}

Eine Anämie kann eine Dyspnoe und Müdigkeit verursachen oder verstärken, ein erhöhter Hämatokrit kann darauf hinweisen, dass die Dyspnoe auf eine primäre Lungenerkrankung zurückzuführen ist. Die Bestimmung von SerumHarnstoff und Kreatinin kann differentialdiagnostische Hinweise auf die Ursache der Volumenüberlastung, primäre Nierenerkrankung oder Herzinsuffizienz geben und ist für die Behandlung von Bedeutung. Eine schwere Hyponatriämie bei einer chronischen Herzinsuffizienz weist auf eine schlechte Prognose hin, und die Erhöhung der Lebertransaminasen kann mit einer Stauung im Splanchnikusgebiet vereinbar sein. Ein Urinstatus ist erforderlich zur Bestimmung einer allfälligen Proteinurie und Glycosurie. Ein infektiöses Geschehen, eine Hypothyreose sowie eine Hyperthyreose, letztere häufig verbunden mit einem tachykarden Vorhofflimmern, sollten als Ursachen der Herzinsuffizienz ausgeschlossen werden. Ein erhöhtes Ferritin kann auf eine Hämochromatose als Ursache der Herzinsuffizienz hinweisen.

\section{Echokardiographie}

Die Echokardiographie ist diagnostisch von hoher Aussagekraft und sollte bei allen Patienten mit Verdacht auf Herzinsuffizienz durchgeführt werden. Über die Beurteilung der systolischen und diastolischen Funktion hinaus liefert sie Informationen über den Zustand der Herzklappen, Grösse der einzelnen Herzkammern und allfällige regionale Wandbewegungsstörungen. Die Doppler-Echokardiographie dient zusätzlich zur Erfassung von Druckgradienten bzw. Regurgitationen bei Klappenvitien und der Bestimmung des Schweregrades einer pulmonalen Hypertonie. Die transoesophageale Echokardiographie erlaubt die Beurteilung von Struktur und Funktion des Herzens bei Patienten, bei denen eine transthorakale Echokardiographie technisch schwierig ist.

Mittels Echokardiographie, während oder nach körperlicher Belastung sowie nach pharmakologischem Stress, können Hinweise auf das Vorliegen einer Myokardischämie bzw. eines ischämischen, aber vitalen Myokards («hibernating myocardium») erhalten werden.

\section{Radionuklidventrikulographie und Perfusionsszintigraphie}

Die mittels Radioisotopen durchgeführte Radionuklidventrikulographie erlaubt die Bestimmung der globalen links- (und rechts-)ventrikulären Funktion, und die Myokardperfusionszintigraphie gibt Aufschluss über die myokardiale Perfusion. Die Technik erlaubt Aussagen bei Patienten, bei denen eine Echokardiographie nicht möglich ist. Die szintigraphische Bestimmung der Myokardperfusion in Ruhe und unter Belastung gibt Hinweise auf das Vorliegen und 
das Ausmass einer Myokardischämie. Neuere Techniken (Positron-Emissions-Tomographie = PET) erlauben zudem, chronisch ischämisches, aber vitales Myokard («hibernating myocardium») zu erkennen. Der Nachteil dieser Methode ist ihre geringe Verbreitung, die hohen Kosten sowie die fehlende Aussage über Herzklappen, Myokard und Perikard.

\section{Kardiale Magnetresonanztomographie}

Am besten reproduzierbare, nichtinvasive Methode zur Erfassung der kardialen Anatomie, Struktur und Funktion. Mittels spezieller Sequenzen (myokardiales Tagging) kann die 3DFunktion (Rotation, Translation und radiale Verkürzung) des linken und rechten Ventrikels quantitativ erfasst werden. Bestimmung der Myokardperfusion bzw. Viabilität sind heute mittels spezieller Sequenzen möglich.

\section{Belastungstest / Spiroergometrie}

Eine normale Leistungsfähigkeit bei nicht behandelten Patienten schliesst eine Herzinsuffizienz weitgehend aus. Bei Patienten mit bekannter Herzinsuffizienz ist der Belastungstest eine Möglichkeit, den Schweregrad der Erkrankung und den Verlauf zu überwachen. Die Bestimmung der maximalen Sauerstoffaufnahme unter Belastung ist einer der besten Parameter, um die Prognose der Erkrankung zu erfassen [3]. Eine $\mathrm{VO}_{2}$ max. unter $10 \mathrm{ml} / \mathrm{kg} / \mathrm{min}$ ist mit einer sehr schlechten Prognose verbunden (Tab. 4).

\section{Invasive Methoden}

Die Herzkatheteruntersuchung zur Druckmessung im Herzen, kleinen und grossen Kreislauf sowie Bestimmung des Herzminutenvolumens und die Koronarangiographie sind zur Diagnosestellung der Herzinsuffizienz in der Regel nicht erforderlich. Da in 60 bis 70\% aller Patienten eine koronare Herzkrankheit Ursache für die Herzinsuffizienz ist, sollte die Indikation zur Koronarographie bei Hinweisen (Anamnese, EKG, Echokardiographie) grosszügig gestellt werden. Sie sollten dann in Erwägung gezogen werden, wenn die Ätiologie der Erkrankung unklar ist und/oder sich aus den dabei erhobenen Befunden therapeutische Konsequenzen ergeben [4]. Eine Endomyokardbiopsie ist gelegentlich zur Diagnostik von infiltrativen oder entzündlichen Erkrankungen nötig.

\section{Neuro-humorale Parameter}

Die Bedeutung neuro-humoraler Mechanismen für die Pathogenese der chronischen Herzinsuffizienz steht ausser Zweifel, hingegen ist der Stellenwert für die Diagnostik der Herzinsuffizienz vorderhand unklar. Entsprechend ist es zurzeit ausserhalb von wissenschaftlichen Untersuchungen nicht sinnvoll, Plasma-Noradrenalin, Renin, Angiotensin-II, Aldosteron, Endothelin oder die verschiedenen natriuretischen Peptide zu bestimmen. «Brain natriuretic peptide» (BNP) verspricht, in der Diagnose der Herzinsuffizenz und zur Abschätzung der Prognose und des Therapieerfolges einige wichtige Hinweise zu geben, die in Zukunft von Bedeutung sein könnten.

\section{Vorgehen in der Praxis}

Bei einem Patienten, der Symptome der Herzinsuffizienz angibt, sollten in der Regel ein EKG, ein Thoraxröntgenbild, ein vollständiges Blutbild, eine Bestimmung der Blutchemie sowie eine Echokardiographie durchgeführt werden. Bleibt die Diagnose zweifelhaft oder wird eine reversible Ursache der chronischen Herzinsuffizienz vermutet, sollten die anderen der oben aufgeführten diagnostischen Tests in Erwägung gezogen werden. Aufgrund der vorliegenden Information sollte es möglich sein, die Ätiologie der Herzinsuffizienz zu bestimmen, Begleiterkrankungen $\mathrm{zu}$ identifizieren, welche den Schweregrad und/oder Verlauf der Herzinsuffizienz mitbeeinflussen, um eine Abschätzung der Prognose vornehmen zu können. Auf diese umfassende Beurteilung des Patienten abgestützt, sollte die angemessene Therapie angesetzt werden.

\section{Therapie der chronischen Herzinsuffizienz}

Die Behandlung der chronischen Herzinsuffizienz hat drei Ziele. Sie soll 1. die Symptome der Patienten vermindern, 2. die Progression der Erkrankung verlangsamen oder aufhalten und 3. die Mortalität senken. Das Management von Patienten mit chronischer Herzinsuffizienz beinhaltet allgemeine Empfehlungen, allgemeine therapeutische Massnahmen, die Pharmakotherapie sowie kathetertechnische und chirurgische Massnahmen [2].

\subsection{Allgemeine Empfehlungen}

\section{Beratung}

Wie bei jeder anderen Erkrankung sollten die Patienten über die Symptome und die Zeichen der Herzinsuffizienz aufgeklärt werden. Ein besseres Verständnis der Erkrankung und ihrer Symptome ist wichtig für die Compliance des Patienten. Besonderer Wert sollte auf die regelmässige Bestimmung des Körpergewichts unter 
standardisierten Bedingungen gelegt werden. Plötzliche Gewichtszunahmen von mehr als $2 \mathrm{~kg}$ in 1 bis 3 Tagen sollten den Patienten dazu veranlassen, medizinischen Rat zu suchen [2]. ${ }^{2}$

\section{Soziale Aktivitäten und Arbeitsfähigkeit}

Wie bei jeder chronischen Erkrankung besteht die Gefahr der sozialen und mentalen Isolation des Patienten. Soziale Aktivitäten sollten deshalb unterstützt werden. Desgleichen sollte der Patient weiterhin seiner Arbeit, in einem Umfang, der seiner physischen Einschränkung angepasst ist, nachgehen.

\section{Flugreisen}

Kürzere Flüge sind wenig belastend. Der Kabinendruck entspricht in der Regel einer Höhe von 1700 bis 1800 m.ü.M., was die meisten Patienten problemlos vertragen. Lange Flüge hingegen können speziell bei Patienten mit fortgeschrittener Symptomatik (NYHA-Klasse III oder IV) zu Problemen führen; diese bestehen in einer möglichen Dehydratation, ausgeprägten Beinödemen und dem Risiko venöser Thrombosen und Embolien. Sofern lange Flugreisen unvermeidbar sind, sollten individuelle Ratschläge bezüglich genügender Flüssigkeitszufuhr, Einnahme von Diuretika und einer Thromboembolie-Prophylaxe (niedermolekulare Heparine) gegeben werden. Patienten sollten auch über die potenziellen Auswirkungen hoher Temperaturen und Luftfeuchtigkeit auf die Flüssigkeitsbalance und den möglicherweise geringeren Diuretikabedarf hingewiesen werden.

\section{Impfungen}

Obwohl keine klinisch kontrollierten Daten über die Effektivität einer routinemässigen Impfung vorhanden sind, sollten Patienten in einem fortgeschrittenen Stadium der Herzinsuffizienz gegen Grippe (1× jährlich) und Pneumokokken geimpft werden.

\section{Empfängnisverhütung}

Eine Schwangerschaft bei Patienten mit fortgeschrittener Herzinsuffizienz (NYHA III-IV) birgt ein grosses mütterliches Risiko. Die Kontrazeption mit Präparaten mit niedrig dosiertem Östrogen und Progesteron (Abkömmlinge der 3. Generation) ist mit einem niedrigen thromboembolischen und Hypertonierisiko behaftet. Intrauterine Spiralen sind eine andere Form der Kontrazeption, ausser bei Patienten mit zugrundeliegenden Klappenerkrankungen, bei denen Infektionen oder eine erforderliche orale Antikoagulation Probleme machen könnten.

\subsection{Allgemeine Massnahmen}

\section{Körperliches Training}

Für kardial stabilisierte Patienten ist ein regelmässiges körperliches Training auf niedrigem Leistungsniveau notwendig. Zu empfehlen sind: dynamische Belastungen (Laufen, Velofahren usw.). Dagegen sollten isometrische Übungen (Gewichtheben usw.) eher vermieden werden. In letzter Zeit weisen viele Daten darauf hin, dass nach optimaler Einstellung der medikamentösen Behandlung ein spezifisches Training zur Verbesserung der körperlichen Leistungsfähigkeit und der Lebensqualität einen mindestens ebenso guten Effekt zeigt wie die zurzeit zur Verfügung stehenden Medikamente. Die Auswirkung auf die Prognose ist wahrscheinlich positiv. Negative Effekte sind bei richtigem Training kaum zu erwarten.

\section{Diät}

Patienten sollten ein normales Gewicht anstreben. Desgleichen sollten bei Patienten mit fortgeschrittener Herzinsuffizienz die Flüssigkeitszufuhr konstant bei 1,5 bis 2 Liter/24 h gehalten werden, ausser in einem warmen Klima. Eine moderate Salzreduktion (2-4 g/24 h) sollte ebenfalls durchgeführt werden.

\section{Rauchen}

Allen Patienten sollte dringend vom Rauchen abgeraten werden.

\section{Alkohol}

Bei Patienten mit Herzinsuffizienz sollte der Alkoholkonsum eingeschränkt werden, da der Alkohol negativ inotrop wirkt und in grösseren Mengen eine akute Dekompensation auslösen kann. Bei Verdacht auf eine äthylische Kardiomyopathie ist eine strikte Abstinenz zu empfehlen. Speziell Vorhofflimmern kann durch Alkohol ausgelöst werden, vor allem wenn eine Herzinsuffizienz vorliegt.

\section{Ruhe}

Bei Patienten mit stabiler chronischer Herzinsuffizienz sind verordnete Ruhephasen nicht sinnvoll. Hingegen ist bei Patienten mit akuter Herzinsuffizienz oder bei fortgeschrittenen Stadien der Herzinsuffizienz Ruhe bis zur Stabilisierung der Symptome empfehlenswert.

\subsection{Pharmakotherapie}

Die medikamentöse Behandlung der Herzinsuffizienz hat in den vergangenen 10 Jahren $\mathrm{zu}$ einer dramatischen Verbesserung der Lebenser- 
wartung (Mortalität) und Lebensqualität (Morbidität) geführt. So konnte z.B. die jährliche Mortalitätsrate von CHF-Patienten der NYHA-Klasse III-IV von $35-40 \%$ auf $12-15 \%$ gesenkt werden. Als «first line drugs» gelten heute die ACEInhibitoren, wobei bei Unverträglichkeit oder Nebenwirkungen mit gutem Erfolg auch die Angiotensin-II-Antagonisten eingesetzt werden können. Als «second line drugs» werden heute die Betablocker eingesetzt, wobei bei diesen das Prinzip «start low, go slow» beachtet werden muss. Als dritter Eckpfeiler der Herzinsuffizienztherapie gelten die Aldosteron-Antagonisten, speziell das Spironolacton [5], welches in der Regel in Kombination mit einem Schleifendiuretikum eingesetzt wird. Die weitere Pharmakotherapie wie Digitalis, positiv inotrope Medikamente oder Antikoagulation - wird im folgenden kurz besprochen.

Angiotensin-converting-Enzym-(ACE-)Inhibitoren ACE-Inhibitoren sind bei allen Patienten mit Herzinsuffizienz, bedingt durch eine systolische Dysfunktion, indiziert, sofern keine absolute Kontraindikation (bilaterale Nierenarterienstenosen, Angioödem während vorheriger ACEHemmer-Behandlung) besteht. Günstige Effekte auf die Progression der Erkrankung sowie ein Trend zur Verbesserung der Mortalität wurde bei Patienten mit asymptomatischer bzw. oligosymptomatischer linksventrikulärer Dysfunktion beschrieben (Evidenz B) [6]. Bei Patienten mit symptomatischer Herzinsuffizienz führen ACEHemmer zu einer Verbesserung der Leistungsfähigkeit $[7,8]$, verlangsamen die Progression der Erkrankung [9] und reduzieren die Mortalität (Evidenz A) [7, 10]. Zudem reduzieren sie bei Patienten mit zugrundeliegender koronarer Herzkrankheit Reinfarkte und das Auftreten einer instabilen Angina pectoris [9]. Günstige Effekte wurden mit verschiedenen ACE-Inhibitoren in einer Reihe von grossen, placebo-kontrollierten und randomisierten Studien gezeigt. Entsprechend darf von einem Klasseneffekt ausgegangen werden. Noch nicht geklärt ist die Frage der Dosierung. Neuere Daten zeigen, dass mit einer hochdosierten ACE-Inhibitortherapie tendenziell bessere Effekte erzielt werden, ohne dass wesentliche Nebenwirkungen in Kauf genommen werden müssen (Evidenz B) [11]. Entsprechend sollte versucht werden, die ACE-Inhibitoren auf die Zieldosen hochzutitrieren, welche in grossen Studien die Mortalität und Morbidität günstig beeinflusst haben (z.B. Captopril $150 \mathrm{mg}$ täglich, Enalapril 40 und Lisinopril 30 mg täglich).

Nebenwirkungen: ACE-Hemmer können zu Hypotonie, Synkopen, Niereninsuffizienz, Hyperkali- ämie und Angioödemen führen. Die häufigste Nebenwirkung ist Husten, welcher manchmal schwer von Husten aufgrund einer pulmonalen Kongestion zu unterscheiden ist. Das Risiko einer relevanten Hypotonie und Niereninsuffizienz steigt bei Patienten mit schwerer Herzinsuffizienz an, insbesondere solchen, welche mit hohen Dosen von Diuretika behandelt werden, bei älteren Patienten und bei Patienten mit einer ausgeprägten Hyponatriämie. Eine arterielle Hypotonie (systolischer Blutdruck < $90 \mathrm{~mm} \mathrm{Hg}$ ) ist akzeptabel, solange der Patient asymptomatisch bleibt und eine stabile Nierenfunktion vorliegt.

\section{Diuretika}

Diuretika sind die Therapie der Wahl bei Patienten mit Zeichen und Symptomen der Flüssigkeitsretention, z.B. Dyspnoe und periphere Ödeme (Evidenz C). Wenn immer möglich sollten Diuretika zusammen mit ACE-Inhibitoren (s. unten) eingesetzt werden.

Schleifendiuretika und Thiazide oder Metolazon haben synergistische Effekte und können in therapierefraktären Fällen in Kombination benutzt werden. Diese Kombinationen führen häufig zu einer ausgeprägten Hypokaliämie, welche kontrolliert und allenfalls substituiert werden muss.

Um Kaliumverluste auszugleichen zu können, sollten niedrig dosierte, kaliumsparende Diuretika eingesetzt werden. Diese sollten zusammen mit ACE-Hemmern nur in kleinen Dosierungen unter initial häufiger Kontrolle des Serum-Kaliums verwendet werden. Die Gabe von Spironolacton zusätzlich zu ACE-Hemmern und anderen Diuretika (bei NYHA-Klasse-IIIund -IV-Patienten) führt zu einer Verbesserung der Prognose [5].

Bei oligosymptomatischen Patienten und erhaltener Nierenfunktion reicht in der Regel die Gabe eines Thiaziddiuretikums aus. Allerdings sind Thiaziddiuretika bei einer glomerulären Filtrationsrate von unter $30 \mathrm{ml} / \mathrm{min}$ wenig effektiv, und bei Patienten mit schwerer Herzinsuffizienz sind meistens Schleifendiuretika erforderlich. Dies kann häufig bei schwerer Herzinsuffizienz und bei älteren Patienten beobachtet werden.

\section{Angiotensin-II-Antagonisten}

Präliminäre Ergebnisse lassen vermuten, dass diesen Medikamenten in Zukunft eine wichtige Rolle bei der chronischen Herzinsuffizienzbehandlung zukommt. So konnte Losartan im Vergleich zu Captopril (Elite-1-Studie) die Mortalität einer älteren Population mit chronischer Herzinsuffizienz (NYHA II-III) signifikant senken [12]. Diese Daten konnten jedoch in der Elite-2- 
Studie nicht bestätigt werden, allerdings waren Losartan und Captopril in bezug auf Mortalitätsreduktion gleich wirksam [13]. Bei Patienten, welche ACE-Inhibitoren nicht tolerieren, sollen diese Medikamente eingesetzt werden, vor allem da sie weniger Nebenwirkungen aufweisen (Evi$\operatorname{denz} C$ ).

Die Resultate der Val-HeFT-Studie zeigen, dass durch die Kombination von Valsartan mit einem ACE-Inhibitor die Gesamtmortalität (primärer Endpunkt) nicht beeinflusst wird. Der zweite primäre Endpunkt aus Gesamtmortalität und Morbidität wurde hingegen um 13,3\% signifikant reduziert. Unter der Kombinationstherapie verminderte sich die Hospitalisationshäufigkeit um 27,5\% und die Lebensqualität verbesserte sich signifikant. Bei Patienten, die keinen ACEHemmer erhielten (ACE-Hemmer-intolerante Patienten) konnte die Mortalität um 44,5\% gesenkt werden (Evidenz B) [14].

\section{Betablocker}

Mehrere placebo-kontrollierte Studien haben gezeigt, dass Betablocker zusätzlich zu ACE-Inhibitoren und Diuretika die Mortalität bei Patienten mit Herzinsuffizienz der Stadien NYHA-Klasse II-IV senken (Evidenz A) [15-18]. Dieser günstige Effekt ist sowohl bei Patienten mit zugrundeliegender ischämischer Herzkrankheit wie auch bei dilatativer Kardiomyopathie vorhanden. $\mathrm{Ob}$ Betablocker mit vasodilatierenden Eigenschaften (z.B. Carvedilol) günstiger sind als kardioselektive Betablocker, bleibt offen und ist Ziel von weiteren Studien.

Die Einleitung der Therapie sollte bei Patienten in einer stabilen Phase der Erkrankung erfolgen und mit sehr kleinen Dosen begonnen werden. Eine vorübergehende Zunahme der Symptomatik ist möglich und kann häufig durch eine Steigerung der Diuretikadosis kompensiert werden. Dosissteigerungen sind in etwa 2- bis 3wöchentlichen Abständen sinnvoll, wobei die maximal tolerierbare Dosis angestrebt werden sollte. Zurzeit sind neben Carvedilol (Zieldosis: $2 \times 25 \mathrm{mg} / \mathrm{d}$ ) [15, 18] Metoprolol (Zieldosis: $2 \times 50$ bis $2 \times 100 \mathrm{mg} / \mathrm{d}$ ) [16] und Bisoprolol (Zieldosis: $10 \mathrm{mg} / \mathrm{d}$ ) [17] zur Behandlung der Herzinsuffizienz registriert.

\section{Hydralazin/Isosorbitdinitrat-Kombination}

Diese Kombination ist eine Alternative zur ACEHemmer-Therapie bei Patienten mit Kontraindikationen bzw. bei Patienten, die ACE-Hemmer nicht tolerieren (Evidenz B) [19]. Diese Kombination wird häufig schlecht ertragen (etwa 30\% Therapieabbrüche wegen Nebenwirkungen).
Nitrate werden häufig bei Patienten mit Angina pectoris eingesetzt, desgleichen werden Nitrate oftmals nachts eingesetzt, um die pulmonale Kongestion und paroxysmale nächtliche Dyspnoe günstig $\mathrm{zu}$ beeinflussen (Preloadreduktion).

\section{Digoxin}

Bei Patienten im Sinusrhythmus bessert Digoxin die Symptome und vermindert die Anzahl der Hospitalisationen (Evidenz B). Desgleichen scheint Digoxin die Zahl der Todesfälle infolge Herzinsuffizienz zu vermindern, dieser Effekt wird jedoch durch eine vermehrte Todesfallrate infolge ventrikulärer Rhythmusstörungen aufgehoben, so dass Digoxin bei Patienten mit Symptomen der NYHA-Klasse II-IV keinen Effekt auf die Gesamtmortalität hat [20].

\section{Aldosteron-Antagonisten}

Die Gabe von Spironolacton zusätzlich zur Basistherapie mit ACE-Hemmern und Schleifendiuretika hat zu einer eindrücklichen Reduktion der Mortalität geführt (Evidenz B) [5]. Neben der ACE-Hemmer- und der Betablockerbehandlung stellt damit der Aldosteron-Antagonist Spironolacton das dritte Medikament dar, welches mit einer signifikanten Verminderung der Mortalität von Herzinsuffizienzpatienten verbunden ist.

\section{Kalzium-Antagonisten}

Kalzium-Antagonisten sollten nicht zur Behandlung der Herzinsuffizienz infolge systolischer Dysfunktion eingesetzt werden. Zweitgeneration-Dihydropyridin-Kalzium-Antagonisten oder Amlodipin bzw. Felodipin können ohne Risiko bei Patienten mit Angina pectoris oder persistierender arterieller Hypertonie eingesetzt werden.

\section{Positiv-inotrope Medikamente}

Sowohl betaadrenerge Agonisten und c-AMPPhosphodiesteraseinhibitoren sind der Behandlung der akuten, therapierefraktären Herzinsuffizienz unter stationären Bedingungen vorbehalten. Die chronische Gabe dieser Medikamente ist in der Regel mit einer gesteigerten Mortalität (Evidenz A) [21, 22] verbunden.

\section{Aspirin}

Niedrig dosiertes Aspirin wird häufig bei Patienten mit zugrundeliegender koronarer Herzkrankheit eingesetzt. Es gibt allerdings keine Daten, die einen günstigen Effekt dieser Therapie auf die Mortalität von Patienten mit Herzinsuffizienz zeigen. 


\section{Orale Antikoagulation}

Die orale Antikoagulation soll das Risiko systemischer Embolien bei Patienten mit fortgeschrittener Herzinsuffizienz und schlechter Ventrikelfunktion vermindern helfen. Kontrollierte Daten dazu existieren nicht. Empfohlen wird die orale Antikoagulation bei Patienten mit Vorhofflimmern, einer Anamnese von systemischen Thrombo- oder Lungenembolien, mit nachgewiesenen endokardialen Thromben, bei linksventrikulären Aneurysmata oder stark eingeschränkter LV-Funktion (EF <30\%) (Evidenz C).

\section{Antiarrhythmika}

Obwohl etwa die Hälfte der Todesfälle bei Patienten mit Herzinsuffizienz auf tachykarde ventrikuläre Rhythmusstörungen zurückzuführen ist, hat die prophylaktische Gabe von Antiarrhythmika entweder keinen oder einen negativen (Klasse-I-Antiarrhythmika) Effekt auf die Mortalität gezeigt. Amiodarone und die Betablocker sind die einzigen Antiarrhythmika, die Patienten mit fortgeschrittener Herzinsuffizienz verabreicht werden können und die einen günstigen Effekt auf die Rhythmusstörungen aufweisen (Evidenz B) [23]. Indikationen für Amiodarone sind Vorhofflimmern (Konversion in und Erhaltung des Sinusrhythmus), symptomatische ventrikuläre Rhythmusstörungen und als zusätzliche Therapie nach Implantation eines implantierbaren Cardioverter-Defibrillators (s. unten).

\subsection{Chirurgische Massnahmen}

\section{Revaskularisation}

Da die koronare Herzkrankheit die häufigste Ursache der chronischen Herzinsuffizienz ist, kommen Revaskularisationsmassnahmen (kathetertechnisch oder Bypassoperation) eine potentiell grosse Bedeutung zu. Bei Patienten ohne Angina pectoris sollte vor einer Revaskularisation ein Vitalitätsnachweis geführt werden, dass hibernierendes, aber vitales Myokard vorhanden ist, das sich nach einer Revaskularisation potentiell erholen kann (Evidenz C). Revaskularisationen bei asymptomatischen Patienten ohne «hibernating» Myokard tragen ein hohes Mortalitätsrisiko, und die Auswirkungen auf die Mortalität im Langzeitverlauf sind unklar.

Implantierbare Cardioverter-Defibrillatoren (ICD) Bei Patienten mit symptomatischen ventrikulären Tachykardien oder Patienten, die wegen eines Kammerflimmerns reanimiert werden mussten, verbessert der implantierbare Cardioverter-Defibrillator die Prognose (Evidenz A) [24].
Zur Reduktion von zu häufigen Entladungen des ICD, welches vom Patienten oft als unangenehm empfunden wird, sollte den Patienten zusätzlich ein Betablocker oder Amiodarone verabreicht werden. Ob die prophylaktische Implantation eines ICD bei asymptomatischen Patienten mit ventrikulären Rhythmusstörungen die Prognose verbessert, ist unklar.

\section{Resynchronisation}

Biventrikuläres Pacing (Elektrode in der Vena cordis posterolateralis und im rechten Ventrikel) zur Verkürzung der QRS-Dauer kann zu einer Verbesserung der klinischen Symptomatik und der linksventrikulären Funktion führen. Diese Therapie wird bei Patienten mit einer QRS-Dauer $>130 \mathrm{~ms}$ angewendet. Verschiedene klinische Studien haben einen günstigen Effekt auf die Lebensqualität und die Hospitalisationshäufigkeit gezeigt (Evidenz B).

\section{Herztransplantation}

Die Herztransplantation sollte in Erwägung gezogen werden, wenn bei Patienten mit schwerer Herzinsuffizienz und einem biologischen Alter $<65$ Jahren alle medikamentösen Massnahmen ausgeschöpft sind und eine andere chirurgische Therapie nicht in Frage kommt (Evidenz A).

\subsection{Auswahl und Zeitpunkt der medikamentösen Therapie}

Asymptomatische linksventrikuläre Dysfunktion Wenn die linksventrikuläre Auswurffraktion $<45-50 \%$ beträgt, ist eine ACE-Inhibitor-Therapie indiziert [6].

\section{Symptomatische, systolische linksventrikuläre Dysfunktion}

Sofern die Patienten keine Zeichen der Volumenüberlastung haben, kann die Therapie mit einem ACE-Hemmer alleine begonnen werden. Wenn eine Besserung der Symptome erreicht wird, sollte die Dosis gesteigert werden. Häufig muss allerdings ein Diuretikum hinzugefügt werden. Aufgrund von neueren Untersuchungen sollten diese Patienten einen Betablocker erhalten [15-18] und zusätzlich niedrig dosiert Spironolacton [5]. Wenn die Patienten symptomatisch bleiben, kann Digoxin zur Verbesserung der Beschwerden hinzugefügt werden [20]. Bei chirurgisch korrigierbaren Faktoren (z.B. linksventrikuläres Aneurysma) sollten entsprechende chirurgische Massnahmen in Erwägung gezogen werden. 
Terminale Herzinsuffizienz (NYHA-Klasse III und IV) Patienten, die trotz ausgebauter medikamentöser Therapie weiterhin symptomatisch bleiben und bei denen herkömmliche chirurgische Eingriffe keine Besserung versprechen, sollten für eine Herztransplantation evaluiert werden. Intermittierende Therapie mit inotropen Substanzen kann dabei erforderlich werden. Desgleichen sind ventrikuläre Assist-Devices bei einigen Patienten als Überbrückung zur Herztransplantation in Erwägung zu ziehen.

\section{Behandlung der Herzinsuffizienz infolge diastolischer Dysfunktion}

Die Herzinsuffizienz auf dem Boden einer alleinigen diastolischen Dysfunktion ist bei jungen Patienten selten; bei älteren kann sie jedoch $30-40 \%$ aller Patienten mit Herzinsuffizienz ausmachen. Häufig ist sie mit einer systolischen Dysfunktion vergesellschaftet. Die Behandlung ist gelegentlich schwierig, da diese Patienten auf Diuretika sehr sensibel reagieren.

Die potentiellen Ursachen einer diastolischen Dysfunktion (Linkshypertrophie bei Hypertonie, Myokardischämie und myokardiale/perikardiale Erkrankungen) sollten gesucht und ursächlich behandelt werden. Tachyarrhythmien sollten konsequent behandelt werden und die Etablierung eines stabilen Sinusrhythmus ist wenn immer möglich anzustreben. Die Gabe eines Betablockers allein oder in Kombination mit einem Kalziumantagonisten ist bei den meisten Patienten sinnvoll. Nitrate können zur Behandlung einer Ischämie eingesetzt werden, eine zu ausgeprägte Vorlastsenkung kann sich jedoch negativ auswirken. Desgleichen ist eine diuretische Therapie immer mit kleinen Dosen zu initiieren. Der Wert einer ACE-Hemmer-Therapie ist unklar, sofern nicht eine Hypertonie besteht. Digoxin ist in der Regel nicht indiziert. 


\section{Literatur}

1 Zbinden S, Hess OM, Mohacsi PJ. Schweizerisches Herzinsuffizienz-Register: Daten aus einer sechsmonatigen Pilotphase. Kardiovask Med 2000; 3(Suppl 2):46S.

2 Remme WJ, Swedberg K; Task Force for the Diagnosis and Treatment of Chronic Heart Failure of the European Society of Cardiology. Guidelines for the diagnosis and treatment of chronic heart failure. Eur Heart J 2001;22:1527-60.

3 Weber KT, Janicki JS. Cardiopulmonary exercise testing for evaluation of chronic cardiac failure. Am J Cardiol 1985;55:22A-31A.

4 Hess OM, Simon RW (Hrsg.). Herzkatheter Einsatz in Diagnostik und Therapie. Heidelberg: Springer Verlag; 1999.

5 Pitt B, Zannad F, Remme WJ, et al. The effect of spironolactone on morbidity and mortality in patients with severe heart failure. N Engl J Med 1999;341:709.

6 The SOLVD Investigators. Effect of enalapril on mortality and the development of heart failure in asymptomatic patients with reduced left ventricular ejection fractions. N Engl J Med 1992;327:685

7 The Captopril-Digoxin Multicenter Research Group. Comparative effects of therapy with captopril and digoxin in patients with mild to moderate heart failure. JAMA 1988;259:539.

8 Kiowski W, Sütsch G, Dössegger L. Clinical benefit of angiotensin-converting enzyme inhibitors in chronic heart failure. J Cardiovasc Pharmacol 1996;27:S19.

9 Konstam MA, Kronenberg MW, Rousseau MF, et al. Effects of the angiotensin converting enzyme inhibitor enalapril on the long-term progression of left ventricular dilatation in patients with asymptomatic systolic dysfunction. SOLVD (Studies of Left Ventricular Dysfunction) Investigators. Circulation 1993;88:2277.

10 The SOLVD Investigators. Effect of enalapril on survival in patients with reduced left ventricular ejection fractions and congestive heart failure. N Engl J Med 1991;325:293.

11 Ryden L, Armstrong PW, Cleland JG, Horowitz JD, Massie BM, Packer M, Poole-Wilson PA. Efficacy and safety of high-dose lisinopril in chronic heart failure patients at high cardiovascular risk, including those with diabetes mellitus. Results from the ATLAS trial. Eur Heart J 2000;21:1967.

12 Pitt B, Segal R, Martinez FA, et al. Randomised trial of losartan versus captopril in patients over 65 with heart failure (Evaluation of Losartan in the Elderly Study, ELITE). Lancet 1997;349:747.
13 Pitt B, Poole-Wilson PA, Segal R, et al. Effect of losartan compared with captopril on mortality in patients with symptomatic heart failure: randomised trial - the Losartan Heart Failure Survival Study ELITE II. Lancet 2000;355(9215): 1582-7.

14 Cohn JN, Tognoni G, for the Val-HeFT investigators. Val-HeFT (Valsartan in Heart Failure Trial). Circulation 2000;102:2672B (abstract).

15 Packer M, Bristow MR, Cohn JN, et al. The effect of carvedilol on morbidity and mortality in patients with chronic heart failure. U.S. Carvedilol Heart Failure Study Group. N Engl J Med 1996; 334:1349.

16 MERIT-HF Study Group. Effect of metoprolol $\mathrm{CR} / \mathrm{XL}$ in chronic heart failure: Metoprolol CR/XL Randomised Intervention Trial in congestive heart failure (MERIT-HF). Lancet 1999;353:2001.

17 CIBIS-II Investigators and Committees. The cardiac insufficiency bisoprolol study II (CIBIS II): a randomised trial. Lancet 1999;353:9.

18 Packer M, Coats AJS, Fowler MB, et al. Effect of carvedilol on the survival of patients with severe chronic heart failure. Carvedilol Prospective Randomized Cumulative Survival, COPERNICUS Study Group. N Engl J Med 2001;344(22):1651-8.

19 Cohn JN, Archibald DG, Ziesche S, et al. Effect of vasodilator therapy on mortality in chronic congestive heart failure. Results of a Veterans Administration Cooperative Study. N Engl J Med 1986;314:1547.

20 Digitalis Investigator Group. The effect of digoxin on mortality and morbidity in patients with heart failure. N Engl J Med 1997;336:525.

21 The Xamoterol in Severe Heart Failure Study Group. Xamoterol in severe heart failure. Lancet 1990;336:1.

22 Packer M, Carver JR, Rodeheffer RJ, et al. Effect of oral milrinone on mortality in severe chronic heart failure. The PROMISE Study Research Group. N Engl J Med 1991;325:1468.

23 Singh SN, Fletcher RD, Fisher SG, et al. Amiodarone in patients with congestive heart failure and asymptomatic ventricular arrhythmia. Survival trial of arrhythmic therapy in congestive heart failure. N Engl J Med 1995;333:77.

24 Moss AJ, Hall WJ, Cannom DS, et al. Improved survival with an implanted defibrillator in patients with coronary disease at high risk for ventricular arrhythmia. Multicenter Automatic Defibrillator Implantation Trial Investigators. N Engl J Med 1996;335:1993. 